\title{
Spectra of nearby galaxies measured with a new very broadband receiver
}

\author{
Gopal Narayanan ${ }^{1,2}$, Ronald L. Snell ${ }^{1}$, Neal R. Erickson ${ }^{1}$, Aeree \\ Chung ${ }^{1}$, Mark H. Heyer ${ }^{1}$, Min Yun ${ }^{1}$, and William M. Irvine ${ }^{1,3}$ \\ ${ }^{1}$ Astronomy Department, University of Massachusetts, Amherst, MA 01003 USA \\ ${ }^{2}$ email: gopal@astro.umass.edu ${ }^{3}$ The Goddard Center for Astrobiology
}

\begin{abstract}
Three-millimeter-wavelength spectra of a number of nearby galaxies have been obtained at the Five College Radio Astronomy Observatory (FCRAO) using a new, very broadband receiver. This instrument, which we call the Redshift Search Receiver, has an instantaneous bandwidth of $36 \mathrm{GHz}$ and operates from 74 to $110.5 \mathrm{GHz}$. The receiver has been built at UMass/FCRAO to be part of the initial instrumentation for the Large Millimeter Telescope (LMT) and is intended primarily for determination of the redshift of distant, dust-obscured galaxies. It is being tested on the FCRAO $14 \mathrm{~m}$ by measuring the $3 \mathrm{~mm}$ spectra of a number of nearby galaxies. There are interesting differences in the chemistry of these galaxies.
\end{abstract}

Keywords. Instrumentation: spectrographs, techniques: spectroscopic, galaxies: ISM

\section{Introduction}

The Large Millimeter Telescope (LMT) is a 50-meter diameter millimeter-wavelength single-dish telescope being built jointly by the University of Massachusetts, Amherst in the USA and the Instituto Nacional de Astrofísica, Óptica y Electrónica in Mexico (Perez-Grovas et al. 2006). The telescope uses recent advances in structural design and active control of surface elements, and aims to reach an overall effective surface accuracy of $\sim 70 \mu \mathrm{m}$ and an ultimate pointing accuracy of better than $1^{\prime \prime}$. The LMT is sited at $4600 \mathrm{~m}$ elevation at a latitude of $19^{\circ} \mathrm{N}$ in the Mexican state of Puebla and offers good sky coverage of both hemispheres. The normally low humidity will allow operation at frequencies as high as $345 \mathrm{GHz}$. Telescope construction is well advanced. Three of the planned five rings of surface panels are in place. The initial complement of instruments will include SEQUOIA, a 32 element heterodyne focal plane array for $3 \mathrm{~mm}$ that is currently in use at FCRAO; AzTEC, a large format, focal plane bolometer array that has had successful runs on the JCMT and ASTE; a dual-polarization receiver for the $1 \mathrm{~mm}$ band; and a unique wide band receiver and spectrometer, the Redshift Search Receiver (RSR), the instrument utilized in the present paper.

The RSR (Erickson et al. 2007) utilizes very wideband indium phosphide MMIC amplifiers operated at $20 \mathrm{~K}$ and has two dual polarization beams (thus a total of 4 receivers). Heterodyne receivers require a fast beam switch to produce flat spectral baselines over such a wide bandwidth, and the RSR uses a novel polarization switch operating at $1 \mathrm{kHz}$ (Erickson \& Grosslein 2007). This is the first wide band, low loss electrical switch operating at a wavelength as short as $3 \mathrm{~mm}$. The fast polarization switch is followed by a broadband orthomode transducer (OMT) that splits the polarizations into two independent receivers. One beam of each polarization of the RSR is always on source.

The principal motivation for the construction of the RSR is to measure the spectra, and particularly the redshift $\mathbf{z}$, of very distant galaxies. Galaxies are believed to form 
in the very early universe with the first episode of star formation $\sim 10^{9}$ years after the Big Bang, corresponding to $(z \gtrsim 6)$. Understanding the formation process requires a catalog of a significant population out to $\mathrm{z} \sim 10\left(5 \times 10^{8} \mathrm{yrs}\right)$. Although these objects are relatively easy to detect in continuum, their distance and age are not easy to determine.

A few of these galaxies have redshifts measured in the visible, but most have no visible counterpart. The strongest emission lines from galaxies at roughly mm-wavelengths are the rotational transitions of $\mathrm{CO}$, and the RSR bandwidth is large enough that there is a very high probability that one line of $\mathrm{CO}$ will fall in the observing band for redshifts $z>1$, and that two CO rotational lines will be in the band for $z>3.2$. When two lines of the CO rotational ladder are detected within the RSR band, the redshift is uniquely determined.

Since the LMT is not yet complete (we are hoping for initial $3 \mathrm{~mm}$ commissioning during 2008), the RSR was installed on the FCRAO $14 \mathrm{~m}$ telescope during spring 2007. The receiver frontend worked very well with the spectrometer to give very flat baselines. CO emission from 22 ULIRGs at moderate redshift was detected (Chung et al. 2008), and broadband $3 \mathrm{~mm}$ spectra of many nearby galaxies were obtained. The latter are presented here.

\section{Observations}

Each pixel of the RSR is sent to 6 different backend cards, each of which can process $\sim 6.5 \mathrm{GHz}$ of bandwidth. Twenty-four backend cards are required to process all four pixels over the $75-111 \mathrm{GHz}$ bandwidth. While all four frontend pixels were available for the Spring 2007 run, only 4 out of the required 24 sections of the RSR backend spectrometer had been fabricated at that time, and were ready to use. For the nearby galaxy work, these four spectrometer cards were hooked up to the best pixel of the frontend to cover first a bandwidth range of $85-111 \mathrm{GHz}$, and then in separate observations a frequency range of $75-92 \mathrm{GHz}$. The two sets of observations are combined and averaged together (using special-purpose software written specifically for the RSR) to produce the spectra presented here.

\section{Results and Discussion}

In Figures 1 and 2, we show the full $3 \mathrm{~mm}$ band spectra towards two galaxies, M82 and IC342. The emission lines from a number of molecular species are detected in the observed galaxies (see Table 1 ). Note that the $J=1-0$ transition of ${ }^{12} \mathrm{CO}$, normally the strongest $3 \mathrm{~mm}$ emission line from galaxies, is not included in the observed bandwidth, but would move into the band for moderate values of the redshift $\mathrm{z}$. As has been found by previous observers (e.g., Aalto 2006, Aalto 2007, Martin 2008), the ratios of the integrated intensities of such important chemical tracers as $\mathrm{HCN}, \mathrm{HNC}, \mathrm{HCO}^{+}, \mathrm{N}_{2} \mathrm{H}^{+}$, $\mathrm{CS}$ and ${ }^{13} \mathrm{CO}$ vary among the galaxies. It has been proposed that such variations may be due to differences in the relative importance of photon-dominated regions (PDRs); $\mathrm{X}$-ray dominated regions (XDRs), perhaps in the vicinity of AGNs; shocked regions; warm, dense molecular clouds; and infrared pumping for $\mathrm{HCN}$ and $\mathrm{HNC}$ (e.g., Aalto 2008, Imanishi et al. 2007, Perez-Beaupuits et al. 2007, Turner \& Meier 2008). Because of the rather large beam-size of the FCRAO $14 \mathrm{~m}$ telescope (from $45-65$ arcsec), it is difficult to definitely separate these effects for our galaxies; this will be much more clear cut with the LMT, whose corresponding beam size will be in the range $12-18$ arcsec. Nonetheless, we note that our results for the ratios of the integrated intensities 


\begin{tabular}{l|l|l|l|l}
\hline Ratio & NGC 253 & M 82 & IC342 & Arp 220 \\
\hline HNC /HCN & 0.51 & 0.43 & 0.37 & 0.81 \\
& $(0.03)$ & $(0.02)$ & $(0.06)$ & $(0.11)$ \\
$\mathbf{H C O}^{+} / \mathbf{H C N}$ & 0.84 & 1.47 & 0.64 & 0.50 \\
& $(0.03)$ & $(0.04)$ & $(0.07)$ & $(0.11)$ \\
$\mathbf{H C N} /{ }^{13} \mathbf{C O}$ & 0.92 & 0.87 & 0.55 & 1.8 \\
& $(0.03)$ & $(0.03)$ & $(0.03)$ & $(0.6)$ \\
$\mathbf{C S} / \mathbf{H C N}$ & 0.43 & 0.58 & 0.33 & 0.55 \\
& $(0.02)$ & $(0.03)$ & $(0.05)$ & $(0.14)$ \\
$\mathbf{N}_{2} \mathbf{H}^{+} / \mathbf{H C N}$ & 0.06 & $<0.02$ & 0.24 & 0.45 \\
& $(0.02)$ & & $(0.07)$ & $(0.08)$ \\
\hline
\end{tabular}

\begin{tabular}{|c|c|c|}
\hline Line & | Frequency (GHz) & Transition \\
\hline $\mathrm{C}^{18} \mathrm{O}$ & 109.8 & $1-0$ \\
\hline $\mathrm{HC}_{3} \mathrm{~N}$ & 109.1 & $12-11$ \\
\hline $\mathrm{CH}_{3} \mathrm{C}_{2} \mathrm{H}$ & 102.5 & $6(\mathrm{n})-5(\mathrm{n})$ \\
\hline $\mathrm{HC}_{3} \mathrm{~N}$ & 100.1 & $11-10$ \\
\hline $\mathrm{SO}$ & 99.3 & $3(2)-2(1)$ \\
\hline $\mathrm{CH}_{3} \mathrm{OH}$ & 96.7 & $\begin{array}{l}2(0,2)-1(0,1) \\
A+, \& \text { blend }\end{array}$ \\
\hline $\mathrm{C}_{2} \mathrm{H}$ & 87.3 & $1-0,3 / 2-1 / 2$ \\
\hline $\mathrm{CH}_{3} \mathrm{C}_{2} \mathrm{H}$ & 85.5 & $5(\mathrm{n})-4(\mathrm{n})$ \\
\hline $\mathrm{C}_{3} \mathrm{H}_{2}$ & 85.3 & $2(1,2)-1(0,1)$ \\
\hline $\mathrm{CH}_{3} \mathrm{OH}$ & 84.5 & $5(-1,5)-4(0,4) \mathrm{E}$ \\
\hline $\mathrm{HC}_{3} \mathrm{~N}$ & 81.9 & $9-8$ \\
\hline $\mathrm{CH}_{3} \mathrm{OH}$ & 81.0 & $7(2,6)-8(1,7) \mathrm{A}-$ \\
\hline
\end{tabular}

Table 1. (a) Molecular Line Ratios in 4 Galaxies $(J=1-0$ except CS, $J=2-1)$. (b) Other detected lines.

$\mathrm{HCO}^{+} / \mathrm{HCN}, \mathrm{HNC} / \mathrm{HCO}^{+}$, and $\mathrm{HNC} / \mathrm{HCN}$ (see Table 1a) agree well for M82, NGC 253 and Arp 220 with those reported by Baan et al. (2008) (observations with the IRAM $30 \mathrm{~m}$ and the SEST $15 \mathrm{~m}$ ), although those latter authors give somewhat higher values for the $\mathrm{HNC} / \mathrm{HCN}$ ratio in NGC 253.

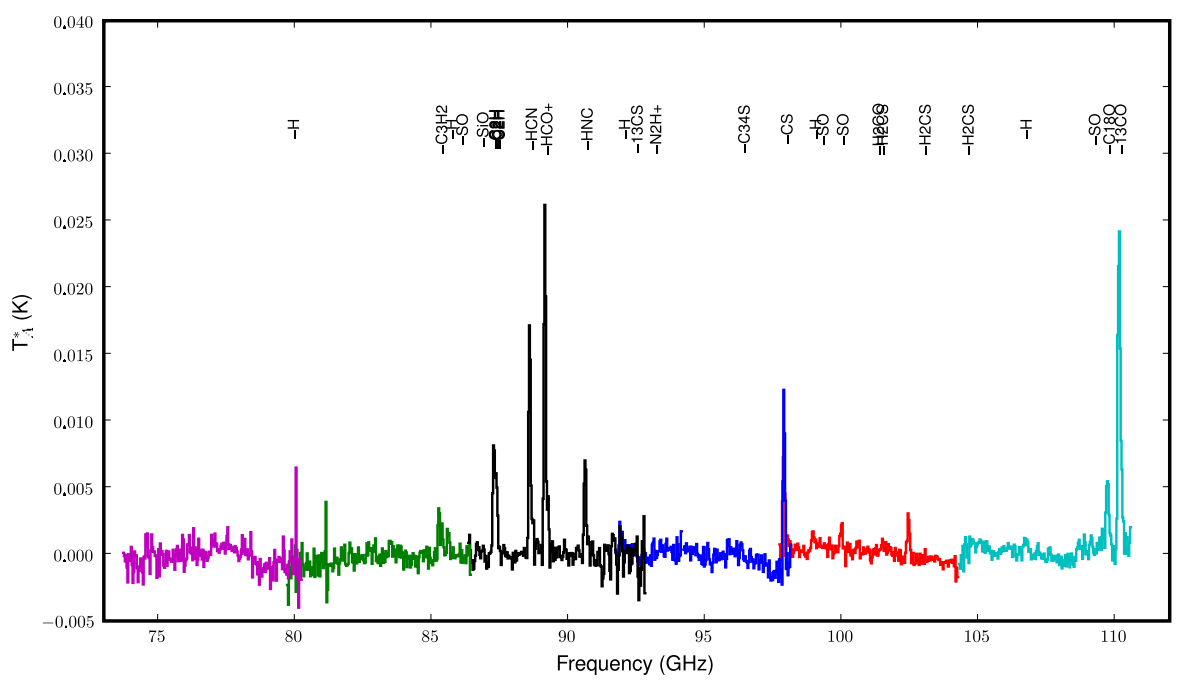

Figure 1. Full $3 \mathrm{~mm}$ band spectrum of M82 obtained with the redshift search receiver. The different colors represent distinct spectrometer boards that were used in the measurment. The names of important molecular lines typically seen in the ISM are denoted at the appropriate frequencies in the plot. M82 has strong continuum emission, and that accounts for the non-flat baselines (compare with Figure 2).

\section{Summary}

Spectra in the 3-mm wavelength band covering 75 to $111 \mathrm{GHz}$ have been observed for about 10 galaxies using a new, very broadband receiver (RSR) with the FCRAO $14 \mathrm{~m}$ radio telescope. Interesting differences in line ratios are found, consistent with previous observations. When the RSR is mounted on the LMT in Mexico, surveys of the chemistry 


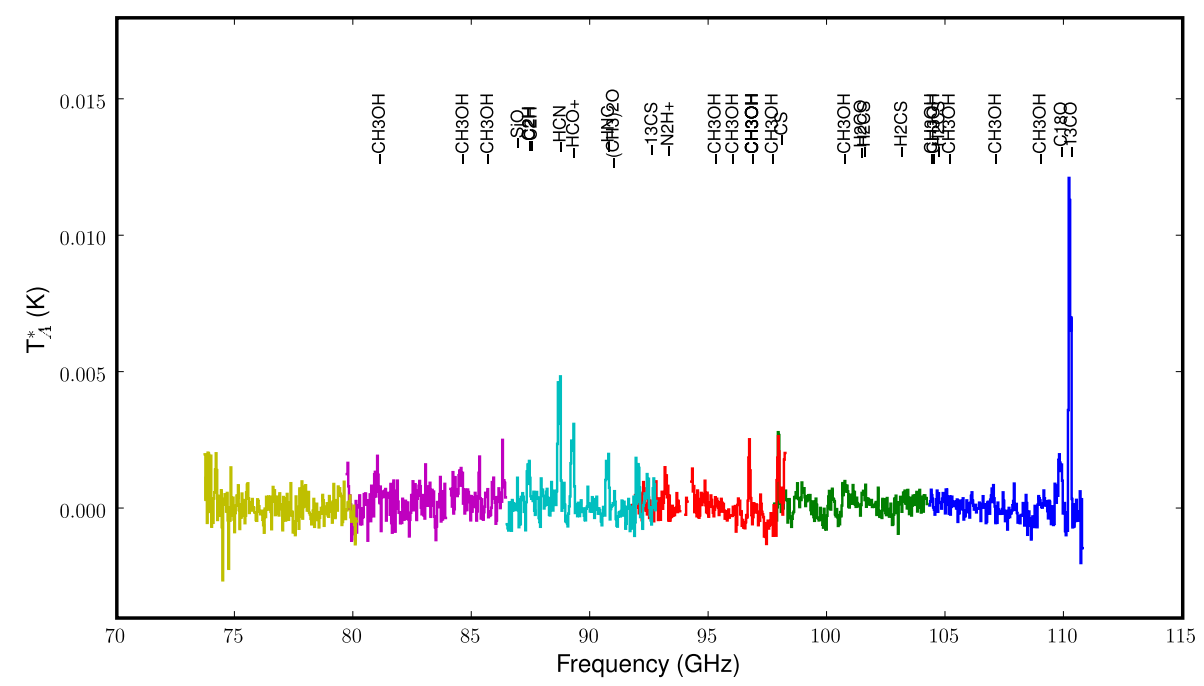

Figure 2. Full $3 \mathrm{~mm}$ band spectrum of IC342 (see Figure 1).

of external galaxies will be possible. We note in this connection the suggestions that the variety of environments near the center of the Milky Way may serve as templates for unraveling the processes in external galaxies (Martin et al. 2008, Jones \& Burton 2008).

\section{Acknowledgements}

We are grateful for partial support of this research by the NSF (AST-0096854 and AST-0704966) and by NASA GSFC Cooperative Agreement NNG04G155A; and to Mike Brewer, Ron Grosslein, Kamal Souccar, Don Lydon and Gary Wallace for assistance during the engineering commissioning of the RSR at the FCRAO $14 \mathrm{~m}$ telescope.

\section{References}

Aalto, S. 2006, Proc. IAU Symposium 231, ed. Lis, D. C., Blake, G. A. and Herbst, E., Cambridge Univ. Press.

Aalto, S. 2007, A\&A A 475, 479

Aalto, S. 2008, ApESSS, 313, 273

Baan, W. A., Henkel, C., Loenen, A. F., Baudry, A., \& Wiklind, T. 2008, A\& A, 477, 747.

Chung, A., Narayanan, G., Yun, M., Heyer, M. H., \& Erickson, N. R. 2008, in prep. ApJ.

Erickson, N. R., Narayanan, G., Goeller, R., \& Grosslein, R. 2007, From Z-Machines to ALMA: (Sub)Millimeter Spectroscopy of Galaxies, 375, 71

Erickson, N. R. \& Grosslein, R. 2007, IEEE Trans. Microwave Theory Tech, 55, 2495.

Imanishi, M., Nakanishi, K., Tamura, Y., Oi, N., \& Kohno, K. 2007, AJ, 134, 2366.

Jones, P. A., \& Burton, M.G. 2008, these Proceedings, in press.

Martin, S., Requena-Torres, M. A., Martin-Pintado, J., \& Mauersberger, R. 2008, Ap\&SSS, 313, 303.

Martin, S. 2008, in: Meech, K., Mumma,M., Siefert, J., and Werthimer, D. (eds.), Bioastronomy 2007: Molecules, Microbes and Extraterrestrial Life, (Astronomical Society of the Pacific)

Perez-Beaupuits, J. P., Aalto, S., \& Gerebro, H. 2007, A $\mathscr{E} A$, 476, 177.

Perez-Grovas, A. S., Schloerb, P. F.,Hughes, D., \& Yun, M. 2006, SPIE, 6267, 1.

Turner, J. L. \& Meier, D. S. 2008, ApESSS, 313, 267. 


\section{Discussion}

Olofsson: How is the site compared to, for instance, Mauna Kea?

IRVINE: It is certainly seasonal. There is a rainy season in the summer, when we would confine ourselves to the $3 \mathrm{~mm}$ region. It is a good site, probably not as good as Mauna Kea. We won't be capable of observing at wavelengths shorter than, say, $0.8 \mathrm{~mm}$.

Ceccarelli: When will we start to operate the telescope?

IRVINE: We are in the process of re-aligning the surface for the inner 3 rings of panels. The telescope had a good appearance in some of those pictures, taken when we had a dedication for President Vicente Fox of Mexico, before he finished his term about a year ago. We hope to begin some $3 \mathrm{~mm}$ tests this year with the inner 3 rings of panels.

Ceccarelli: A scientific question. I saw that the ratio of $\mathrm{N}_{2} \mathrm{H}^{+} / \mathrm{HCN}$ is the largest difference between the galaxies that you showed. Can you comment on this?

IRVINE: No, I really can't. I should point out that the beam size of the $14 \mathrm{~m}$ telescope is pretty large on these galaxies, so we are probably looking at a range of different regions in these galaxies. That makes it a little difficult to interpret.

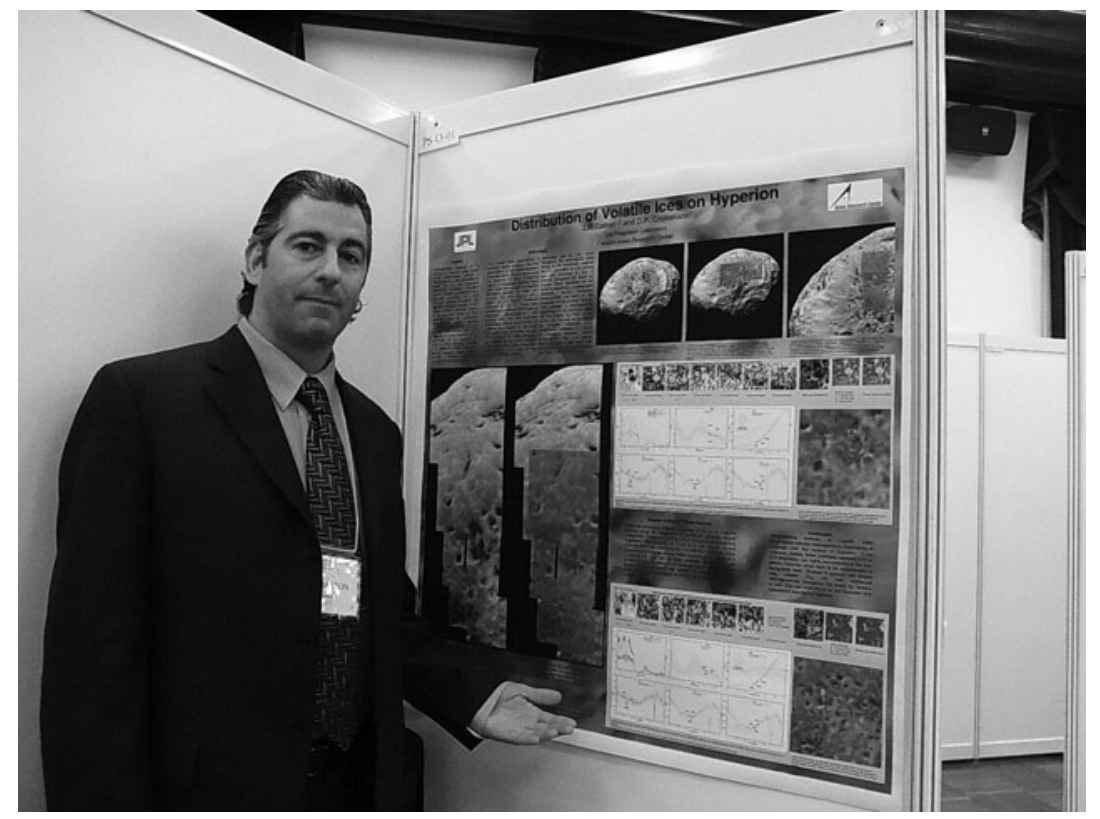

Brad Dalton showing his poster. 


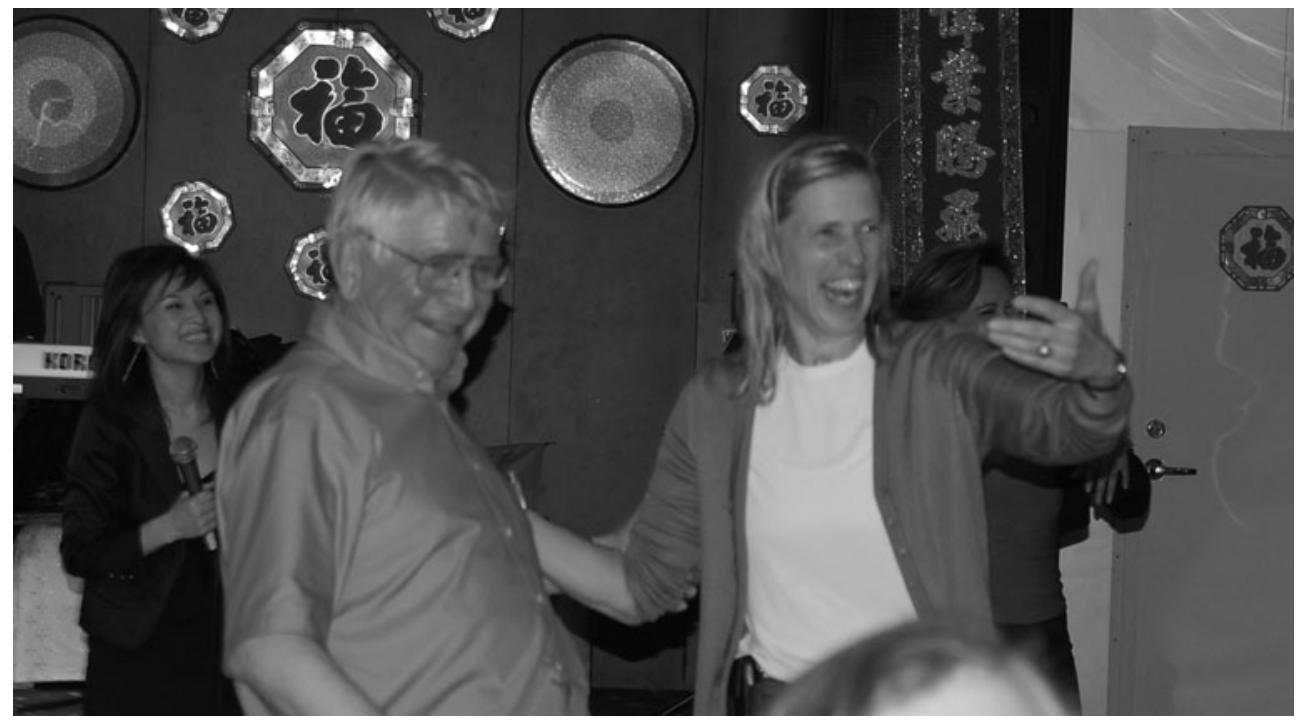

Peter Strittmatter and Lucy Ziurys having fun on the dance floor of the Bauhinia. 